\title{
Node Deployment in Farmland Wireless Sensor Networks based on Renewable Energy
}

\author{
https://doi.org/10.3991/ijoe.v12i10.6210 \\ Jianwei $\mathrm{Wu}{ }^{1,2}$, Yisheng Miao 1,3 \\ ${ }^{1}$ Beijing Academy of Agriculture and Forestry Sciences, Beijing, China \\ 2 Beijing PAIDE Science and Technology Development Co. Ltd, Beijing, China \\ ${ }^{3}$ National Engineering Research Center for Information Technology in Agriculture, Beijing, China
}

\begin{abstract}
Wireless sensor network (WSN) plays an important role in the large scale farmland environmental monitoring. The complex farmland environment has a great impact on the WSN performance. Extremely low power consumption of WSN is required because of the long monitoring period and limited energy. In the considering of network coverage, connectivity, organization and power consumption, this paper proposes a new deployment strategy in the consideration of solar power nodes. A mixed deployment method combining with structure and random filling deployment is used. The hot-spot nodes in the network are replaced by solar nodes in order to get a longer lifetime. The simulation results show that the new method has a better performance than the traditional algorithms.
\end{abstract}

Index Terms-wireless sensor network, agriculture, node deployment, solar power node, energy saving.

\section{INTRODUCTION}

With the development of MEMS (Micro-ElectroMechanical System) and wireless communication technologies[1], WSNs (Wireless Sensor Networks) have played an important role in many fields[2-3]. In agriculture, it is helpful in monitoring the farmland environment with WSNs. In this case, the environment information can be automatically measured, gathered and transmitted to a base station far away. Agriculture workers would use these information to guide and improve the agricultural production. However, there are many restrictions and problems in farmland WSN applications.

One of the most important question is how many nodes should be deployed to the farmland. The connectivity of the network may be bad when there are not enough nodes, or some nodes are not in a suitable place. Increasing the number of nodes often improves network connectivity, but the cost may increase. Some node deployment methods are proposed for this kind of issue.

Limited energy is the bottleneck in the farmland WSN application. So the low power strategies are very important. The low power strategy will decrease the power consumption of each node or average the power consumption between nodes, both of which would lead to a longer network lifetime.

Due to the need of accurate and convenient accessing of farmland environmental information, WSN has become a key technology in the implementation of precision agriculture. In the large scale farmland wireless sensor network monitoring applications, there are many characteristics need to be considered, such as large monitoring area, huge number of nodes, very limited node energy and etc. Node deployment will affect data collection and network lifetime and other properties directly. For all the above reason, how to deploy the nodes effectively becomes a research point.

Existing node deployment researches mainly focused in two parts. One is using minimal nodes to achieve the coverage of the monitoring area. The other is how these nodes located to make sure the wireless sensor network has good performance such as connectivity, lifespan and so on. It is very hard to change node batteries in the farmland application, so the energy limitation is more critical. In recent years, renewable energy is a new idea in dealing with the WSN energy problems. Farmland is rich in solar and wind energy resources, by using these, the WSN energy problems could be solved fundamentally. Because of the high cost of the solar power system, there are only a small part of nodes could using it in the agricultural WSN application.

Considering the feature of large scale farmland heterogeneous network and the solar energy obtaining, this paper proposes NHSND (Network Hot-spot Solar Node Deployment). The scheme reduces the deployment complexity and significantly extending the lifespan of the overall network.

\section{RELATED WORKS}

Node deployment research mainly focuses on the network connectivity and coverage. A reasonable node deployment can offer a good network performance. A redundant deployment may bring better network connectivity and higher coverage rate, but also increases network cost and power consumption. So a key issue in node deployment is to find a balance point between connectivity, coverage, cots and power. The deployment strategy can be classified in different ways, such as structure deployment and random deployment, static deployment and dynamic deployment.

Static deployment is a commonly used method in which the location of nodes is fixed or changed slightly after deployment. A static deployment algorithm focuses on finding an optimization method for large combination of nodes. Many algorithms are proposed for solving this kind of issue, such as Glowworm Swarm Optimization(GSO) algorithm[4], Particle Swarm Optimization (PSO) algorithm[5] and Simulated Annealing (SA) algorithm[6]. All these methods have been find good performances in agriculture WSN applications, especially in large scale network. 
However, when the working environment of the WSN changes, the original deployment may perform badly in connectivity or coverage. And dynamic deployment algorithms are proposed to dealing with it. In dynamic deployment, the node is supposed to have the ability to change its location. After the initial deployment, the nodes could choose to move or not based on the environment change. By change the node's location, the dynamic deployment algorithm can achieve a better performance with lower cost than static method.

Random deployment is the simplest deploying method, but in some cases most useful. In some danger or complex environment, such as the battlefield, special terrain, disaster area, it is unable to place the nodes directly by human. In these cases, random deployment by machines or airdrop will be high efficiency. Li[7] proposed an scheme to estimate the required number of nodes under random deployment. In order to ensure the connectivity and coverage of the wireless sensor network, and lower the network power and cost, the node density $\lambda$ should be

$$
\frac{\sqrt{3}}{6 R_{S}^{2}} \leq \lambda \leq \frac{2 \sqrt{3}}{3 R_{S}^{2}}
$$

Where, $R_{S}$ is the perception radius of the sensor nodes.

However, the random deployment may be insufficient for the uncertain location of nodes. Some nodes may be very close to each other, which will raise network redundancy. And the network performance of the random deployment could not be guaranteed.

Under a controllable environment, the structured deployment could be done. Firstly, the monitoring area is divided according to the topology geometry. The node position is previously calculated and then the sensor nodes are deployed. Liu[8] proposed a systematic regular deployment in patterns such as triangular lattice, square lattice and hexagon lattice, and the maximum lattice side in each pattern was given. The deployment cost and connectivity in these three regular patterns were also discussed. Sun[9] compared random deployment, square deployment and hexagon deployment by simulation. The hexagonal plan was determined as the final deployment method, and experiments were carried out in the fields. Structured deployment enables precise control of node position layout, so the network performance of coverage and connectivity could be optimal. However, due to the need of human intervention, the structured deployment is less efficient than random deployment.

Energy which is the scarcest resource in WSNs determines the lifetime of WSNs[10]. A WSN nodes mainly contain four parts: processing module, sensor module, communication module and power supply module. Most part of energy is consumed by sensing, calculating and communication processes. Especially, nearly a half of the total power consumes during data transmission and receiving[11-13]. In order to maximize the WSN lifetime, many energy saving strategy was developed for the agriculture WSNs.

In order to achieve the goal of energy saving and balancing, the existing algorithms select cluster head nodes based on the nodes residual energy. This kind of strategies may not suit the networks which employ both battery powered nodes and solar powered nodes. That is because the solar powered nodes obtain the energy from the envi- ronment and the residual energy changes with time. The introduction of renewable energy may solve the energy problem fundamentally. Agricultural WSN covers a vast area, and have abundant renewable energy sources such as solar and wind. However, due to the high cost of renewable energy supply system, the agriculture WSN could only have a part of node to be renewable energy supply. In this case, the research would mainly focuses on the mixedpower supply strategy.

\section{SySTEM Model, ASSUMPTION AND PROBLEM DESCRIPTION}

\section{A. Network Assumptions}

The network assumptions are as follows[14-17]:

(1) The network is deployed in an $\mathrm{M} \times \mathrm{M}$ square region;

(2) The transmission radius of all sensor nodes could vary from 0 to $r_{\max }$, where $r_{\max }>r$;

(3) Each source node generates a raw data packet with the same size $l$;

(4) The network has only one sink node(or base station) with infinite energy collects the data from all other nodes;

(5) There two types of nodes in the network. One is battery powered with finite energy, the other is solar powered which has continuous energy input. For assumption, $E_{b}$ refers to the initial power of a battery powered node, and $E_{s}$ refers to the initial power of a solar powered node. $E_{b}$ $<<E_{s}$;

(6) All nodes have the capability of monitoring the energy level of itself;

(7) The network is hierarchical structure organized, with a cluster radius $r$.

\section{B. System Models}

The communication power consumption model is set as in reference ${ }^{[18]}$. The transmitting power $\mathrm{E}_{\mathrm{tx}}$ is:

$$
E_{t x}=\left\{\begin{array}{l}
l E_{\text {elec }}+l \varepsilon_{f} d^{2}, d<d_{\text {crossover }} \\
l E_{\text {elec }}+l \varepsilon_{m} d^{4}, d \geq d_{\text {crossover }}
\end{array}\right.
$$

where, $l$ refers to the number of bits in a packet, $E_{\text {elec }}$ refers to the energy consumption which transmitting circuit or receiving circuit transmit one bit data, $d$ refers to the distance between the transmit and receive nodes, $d_{\text {crossover }}=\sqrt{\varepsilon_{f} / \varepsilon_{m}}$, is a threshold distance in the model, if $d$ is less than $d_{\text {crossover }}$, then the free space attenuation model is used. if $d$ is larger than $d_{\text {crossover }}$, the multi-path attenuation model is used $\varepsilon_{f}$ and $\varepsilon_{m}$ are the energy coefficients of power amplification in two models.

The receiving power $E_{r x}$ is:

$$
E_{r x}=l E_{\text {elec }} .
$$

There are cluster-head nodes and non cluster-head nodes. So the total energy consumption is the sum of cluster heads and non cluster heads. The power consumption of cluster-head node $E_{C H}$ is calculated as:

$$
E_{C H}=l E_{\text {elec }} \frac{N}{k}+l E_{B F} \frac{N}{k}+l \varepsilon_{m} d_{t o B S}^{4} .
$$


PAPER

Node DePLOYMENT IN FARMLAND WIRELESS SENSOR NETWORKS BASED ON RENEWABLE ENERGY

where, $E_{B F}$ refers to the cluster-head's data fusion power per bit information, $d_{t o B S}$ refers to the distance between cluster-head and the base station.

The power consumption of intra-cluster nodes $E_{n C H}$ is calculated as:

$$
E_{n C H}=l E_{\text {elec }} \frac{N}{k}+l \varepsilon_{f} d_{t o C H}^{2}
$$

where, $d_{t o C H}$ refers to the distance between intra-cluster node and cluster-head.

According to the system model, each cluster region is considered as a round area with a cluster as the center. The total monitoring area is $M^{2}$, the optimal cluster number is set to $k$. Intra-cluster nodes distribute evenly in the round area. The expectation of $d^{2}{ }_{t o C H}$ is calculated as:

$$
E\left[d_{t o C H}^{2}\right]=\iint\left(x^{2}+y^{2}\right) \rho d x d y=\frac{M^{2}}{2 \pi k} .
$$

where, $\rho$ refers to the node distribution coefficient, the value of $\rho$ equals the reciprocal of area when the nodes are evenly distributed.

\section{Problem Description}

According to the network models and assumptions, there are high performance and high cost solar powered nodes in the network, so the network overall power consumption and cost should take into consideration as well as network connectivity and coverage. Under the mixed power supply WSNs, there four types of problems need to be considered in the network deployment strategy optimization.

(1) Network connectivity and area coverage, which are the basic requirement of the deployment strategy;

(2) Network cost. The performance would be better if there is more nodes. The problem is to meet the basic performance requirement in a lowest cost;

(3) Node location. Under a certain number of nodes, the nodes location have a huge impact to the network performance and lifetime. The location of each node is the key problem in the deployment.

(4) Number and location of solar powered nodes. Solar nodes have better performance and much more power, but the number of solar nodes should be limited due to the high cost. And their location are also important to the overall performance.

\section{NeTwork Hot-SPot Solar Node DePloyment STRATEGY}

\section{A. Undifferentiated node deployment stage}

In this stage, all nodes is treated as battery node in order to satisfy the network coverage and connectivity[18-20]. The deploy method proposed in this paper is a combination of structure and random filling deployment. The structure deployment could provide an overall coverage of the monitoring area which avoids nodes unbalance distribution. The random filling deployment provides a more operational way for deployment in large-scale farmland environment, which reduces the difficulties in the deployment. The nodes unbalance distribution caused by random deployment can be ignored in a small region.
The number of nodes should be decide according the monitoring area and node coverage area.

$$
N_{a}=C_{r} \frac{A_{m}}{\pi r^{2}} .
$$

where, $N_{a}$ refers to the needed number of nodes, $A_{m}=M \times M$ refers to the monitoring area, $r$ refers to the node sensing radius, $k$ is the coverage factor. The network will have more nodes with a bigger $C_{r}$, which may lead to a higher performance and redundancy. Generally $C_{r}$ takes a value between 1.5 to 2 .

The structure deployment method includes deltoid deployment, square deployment and hexagon deployment. Reference [3] has proved the hexagon deployment is better than square deployment. So the hexagon deployment is used as structure deployment method in the paper. An illustration of mixed deployment is shown in figure 1 .

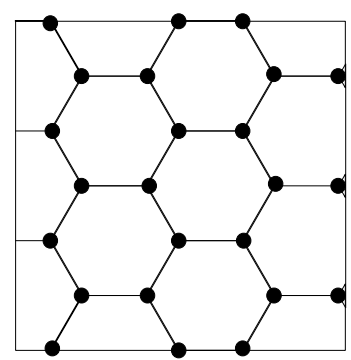

(a)

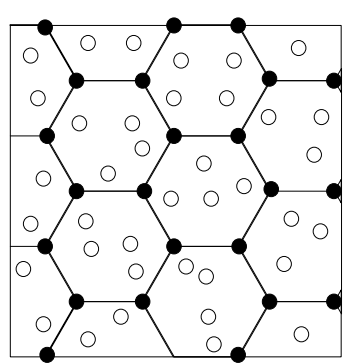

(b)
Figure 1. Steps in mixed deployment

(a) Frame structure deployment (b)Random filling deployment

As it is shown in figure 1(a), a regular hexagon frame structure deployment is used, which is used to meet the basic network coverage requirement. By doing this, the probability of uneven distribution of random deployment is minimized. A random filling deployment in each frame area is done, as it is shown in figure 1(b), which can simply implement and satisfy the network connectivity.

$N_{f}$ is the number of nodes which build the regular hexagon frame.

$$
N_{f}=6 A_{n}=6\left\lfloor\frac{A_{m}}{\sqrt{3} a^{2}}\right\rfloor .
$$

where, $A_{n}=\left\lfloor\frac{A_{m}}{\sqrt{3} a^{2}}\right\rfloor$ refers to the number of regular hexagon area, $a$ refers to the edge length of regular hexagon. The deployment is more randomize when $a$ becomes larger. When $a \leq r$ the mixed deployment becomes a structure deployment. In this paper $a=3 r$.

$N_{c}$ refers to the average number of nodes which filling to the each frame area.

$$
N_{c}=\left\lceil\frac{N_{a}-N_{f}}{A_{n}}\right\rceil .
$$

\section{B. Network hot-spot solar node deployment stage}

In a hierarchical network architecture, cluster head works as "super node" which takes the response of all the data transfer task. The energy consumption of cluster 
heads which consume more power than the other normal nodes is the hot-spot of the network. Solar powered nodes which have continuous power supply are suit for cluster heads. Meanwhile, there is plenty of solar power in the farmland environment which is easy to get use of. The introduce of solar power can prolong the lifespan of farmland wireless sensor network, but also rises the network cost. So the number of solar node need to be optimized in order to get a balance between network lifetime and cost.

After the undifferentiated node deployment stage, the total number of the nodes and the location of nodes are all fixed. In the second stage, the number and location of solar powered node would be decided. In this section, a solar node deployment strategy based on network hot-spot is proposed. The flowchart of network hot-spot solar node deployment algorithm is shown in figure 2.

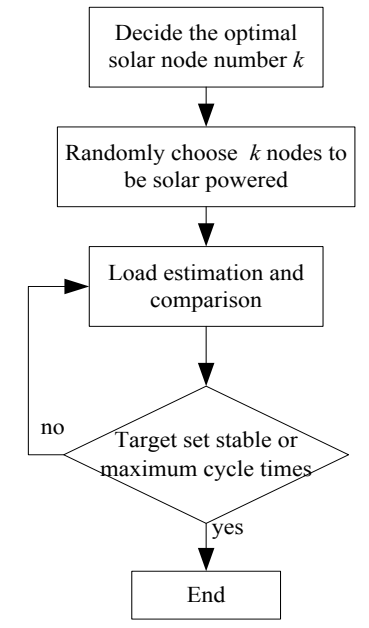

Figure 2. Network hot-spot solar node deployment flowchart

The number of solar nodes equals to the optimal number of clusters $k$. In order to get an optimal $k$, a Linear Programming issue is built. The constrain is to minimize the network overall energy consumption.

$$
\left\{\begin{array}{l}
\frac{d E_{\text {total }}}{d k}=0 \\
\frac{d^{2} E_{\text {total }}}{d k^{2}}>0
\end{array} .\right.
$$

where $E_{\text {total }}$ refers to the overall network power consumption, and $k$ refers to the optimal cluster number.

According to the system models such as equation (4), (5) and (6), the network overall energy consumption is:

$$
\begin{aligned}
& E_{\text {total }}=k\left(E_{C H}+\frac{N}{k} E_{n C H}\right) \\
& =l\left(2 E_{\text {elec }} N+E_{B F} N+k \varepsilon_{m} d_{\text {toBS }}^{4}+N \varepsilon_{f} \frac{M^{2}}{2 \pi k}\right)
\end{aligned}
$$

Lead equation (11) into formula (10), the optimal $k$ could be obtained as.

$$
k=\left\lceil\frac{\sqrt{N}}{\sqrt{2 \pi}} \frac{\sqrt{\varepsilon_{f}}}{\sqrt{\varepsilon_{m}}} \frac{M}{d_{t o B S}^{2}}\right\rceil .
$$

After the number of solar nodes is got, the location of the solar nodes could be decided by the following steps. step 1: Random choose $k$ nodes to be the target nodes. The target nodes set is noted as $\{\mathbf{T N}\}$. For any node $\mathrm{S}_{\mathrm{i}}$, $\mathrm{S}_{\mathrm{j}} \in\{\mathbf{T N}\}$, if $d_{i j} \leq r$, do step 1 again, else go to step 2 . Where, $d_{i j}$ is the distance between node $\mathrm{S}_{\mathrm{i}}$ and $\mathrm{S}_{\mathrm{j}}$, and $r$ is the cluster radius.

step 2: For any $S_{i} \in\{\mathbf{T N}\}, S_{j}$ is a neighbor node of $S_{i}$ and $S_{j} \notin\{\mathbf{T N}\} . N_{i}$ is the number of neighbor nodes of $S_{i}$. $\mathrm{C}_{\mathrm{i}}$ is the common neighbor nodes between $\mathrm{S}_{\mathrm{i}}$ and other nodes in $\{\mathbf{T N}\}, \mathrm{C}_{\mathrm{j}}$ is the common neighbor nodes between $\mathrm{S}_{\mathrm{j}}$ and nodes in $\{\mathbf{T N}\}$ except $\mathrm{S}_{\mathrm{i}}$. If $C_{i}<C_{j}$, add $\mathrm{S}_{\mathrm{j}}$ to the target set and delete $\mathrm{S}_{\mathrm{i}}$.

step 3: If there are several nodes satisfy the $C_{i}<C_{j}$ requirement in step 2, choose the node whose number of neighbor nodes is closed to the average number of nodes in a cluster.

$$
\left|N_{j}-\frac{N}{k}\right|=\min \left\{\left|N_{j}-\frac{N}{k}\right|, \mathrm{L},\left|N_{m}-\frac{N}{k}\right|\right\} \cdot
$$

Add $S_{j}$ to the target set and delete $S_{i}$.

Repeat step 2 and step 3 until there is no node change in the target set, or reach the maximum cycle times.

\section{Simulation AND Results}

\section{A. Network simulation environment}

The simulation was worked out using MATLAB 2012. According to reference [19], network model parameters are shown in table I.

TABLE I.

SIMULATION PARAMETERS

\begin{tabular}{|c|c|}
\hline Parameter & Value \\
\hline Monitoring area & $1000 \mathrm{~m} \times 1000 \mathrm{~m}$ \\
\hline Sink(BS) location & $(500,3000)$ \\
\hline$E_{b}$ & $0.5 \mathrm{~J}$ \\
\hline$E_{S}$ & $5 \mathrm{~J}$ \\
\hline$r$ & $100 \mathrm{~m}$ \\
\hline$\varepsilon_{f}$ & $0.0013 \mathrm{pJ} / \mathrm{b}$ \\
\hline$\varepsilon_{m}$ & $10 \mathrm{pJ} / \mathrm{b} \cdot \mathrm{m}^{-2}$ \\
\hline$E_{t x}, E_{r x}$ & $50 \mathrm{~nJ} / \mathrm{b}$ \\
\hline$l$ & $400 \mathrm{~b}$ \\
\hline Basic coverage requirement & $85 \%$ \\
\hline
\end{tabular}

\section{B. Result and analysis}

To test the validity of the algorithm proposed in the paper, random deployment strategy and GA(Genetic Algorithm) was used as the comparison to the NHSND algorithm. The coverage comparison using these three algorithms with different number of nodes was done.

As it is shown in figure 3 . When the number of nodes equals 50, the coverage of random deployment is about $30 \%$, GA is about $35 \%$, and NHSND is about $38 \%$. When node number below 200, coverage of all three algorithms increases rapidly with the increasing of node number. The coverage of random deployment is about $60 \%$, GA is about $85 \%$, and NHSND is about $85 \%$ with a node number of 200 . When the number of nodes is small, the coverage of random deployment algorithm is lower than the 
PAPER

Node DePloyment In FARMLAND WIRELESS SENSOR NETWORKS BASED ON RENEWABLE ENERGY

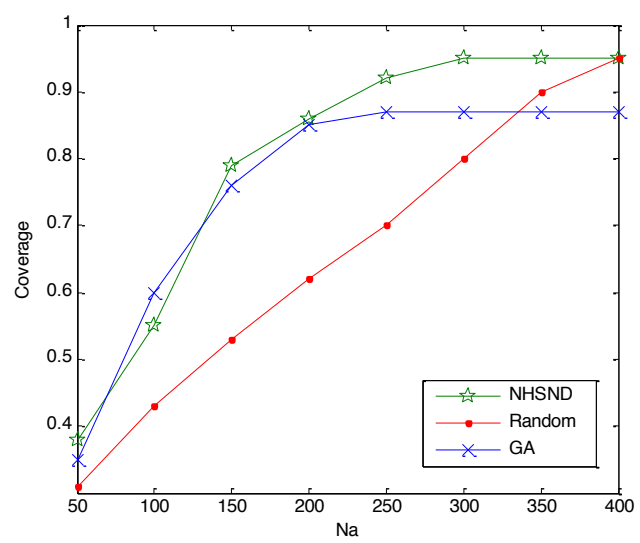

Figure 3. Network coverage comparison

other two methods. Because there may be overlapping between node coverage areas. When the node number goes over 200, the coverage of GA is almost the same, with a final value of about $86 \%$. The coverage of random algorithm keeps increasing when the node number goes over 200, and reaches the highest value of $95 \%$ when the number is 400 . The coverage of NHSND algorithm keeps increasing when the node number goes over 200 to 300 , but the increasing rate is decreased. When the node number is more than 300 , the coverage of NHSND keeps stable at a valve of about $95 \%$.

When the number of nodes gets large, the redundancy of the network gets high, the coverage of random deployment gets to about $95 \%$ in the final state. GA has a good coverage performance when the node number is small. When the node number is larger than 200, the coverage of genetic algorithm keeps below $87 \%$. GA is a local optimization algorithm, which may find a local optimal result instead of a global one. NHSND algorithm has a better performance than the other two in both small and large $N_{a}$ situations. The network coverage of NHSND reaches about $85 \%$ when $N_{a}$ is 200 . NHSND algorithm could reach about $95 \%$ coverage when $N_{a}$ is larger than 300 .

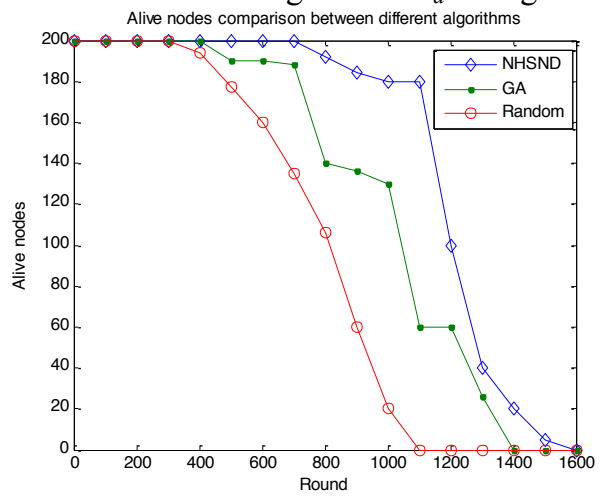

Figure 4. Network lifetime comparison between different algorithms

A performance comparison of NHSND, GA and Random deployment was done. In this comparison, all nodes is set to be battery nodes, and the routing algorithm is CHCS proposed by reference[20]. As it is shown in figure 4 , die nodes appear at about round 400 under random deployment. The nodes in random deployment die at a fixed rate because of the random deployment. Under random deployment, $10 \%$ nodes die at round $500,90 \%$ nodes die at round 1000 , and all nodes die at round 1100 . The results tell that random deployment which has the shortest network lifetime cannot balance node energy consumption very well, and it has the least stable period. In genetic algorithm, die nodes also appear at about round 400 , but the number of alive nodes in genetic algorithm do not decrease as fast as in random deployment. In genetic algorithm, 10\% nodes die at round 700, 90\% nodes die at round 1300 , and all nodes die at round 1400 . The alive nodes number decreases rapidly from round 700 to round 800 , round 1000 to round 1100 , and also round 1200 to round 1400 . In other rounds, the number of alive nodes decrease slowly. That may be because GA is a local optimization algorithm. The deployment of GA may be not so good in some area. Nodes in these areas consume their energy faster than other parts of network. In NHSND algorithm, die nodes appears at round $800,10 \%$ nodes die at round $1100,90 \%$ nodes die at round 1400 , and all nodes goes dead at round 1600 . The network life time of NHSND is about 2 times of random deployment and 1.3 times of GA.

The introducing of solar power is the key point of NHSND, but GA and random deployment algorithm could not handle this situation. The simulation under solar power situation compares different number of solar nodes in NHSND algorithm.

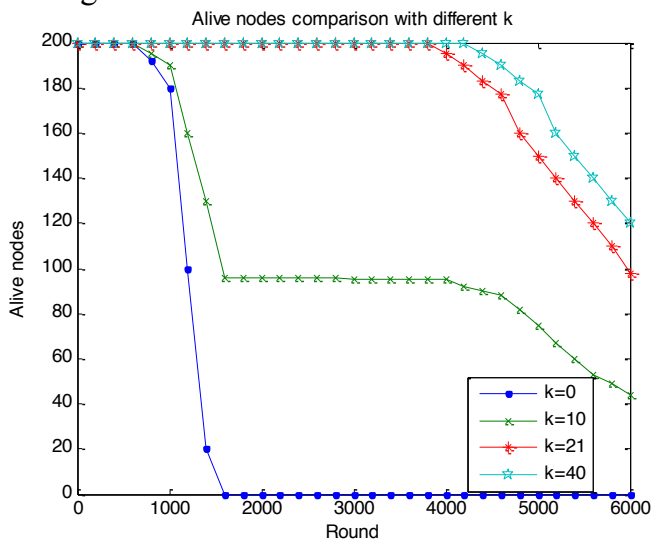

Figure 5. Network lifetime comparison in NHSND with different $k$

As shown in figure 5, when the number of solar nodes $k$ is zero, the result is the same as in figure 4, die nodes appears at round $800,10 \%$ nodes die at round $1100,90 \%$ nodes die at round 1400, and all nodes goes dead at round 1600 . When $k$ equals to 10 , die nodes appear at about round $800,10 \%$ nodes die at round 1000 , and the alive nodes decrease rapidly to about 100 at round 1600 . The reason is that some part of region do not have solar power nodes, so the nodes go dead very quickly. Then the number of alive node do not decrease until round 4000. About half of nodes could find a solar node as a cluster head, so they consume less energy, and work for more than 4000 rounds. After 4000 rounds, the number of alive nodes goes down slowly. The reason may be the nodes which have a distance to the hot-spot exhausted its power. The optimal value of $k$ equals to 21 according to the result in section IV(b). A over solar node supply case of $k$ equals to 40 is used as a comparison. Die nodes appear at about round 4000 in both $k=21$ and $k=40$. In $k=21$ case, $10 \%$ nodes die at round $4800,50 \%$ nodes die at maximum round 6000 . In $k=40$ case, $10 \%$ nodes die at round $5000,40 \%$ nodes die at maximum round 6000 . Meanwhile the decreasing rate of alive node is almost the same in these two cases. The extra 19 solar nodes have only a little contribution to the 
network lifetime according to the result. The simulation results prove the $k$ optimal value's validity.

\section{CONCLUSTION}

Agriculture wireless sensor networks has attracted a lot of attention in the recent years and introduced unique challenges compared to WSN in other applications. In this paper, a new deployment method NHSND is proposed in the consideration of solar power node. A mixed deploy method combining with structure and random filling deployment is proposed to make sure the network coverage and connectivity. A solar power location selection method base on network hot-spot is presented with an optimal number of solar nodes. According to the simulation results, the NHSND algorithm has a better coverage and network life time than genetic algorithm and random deployment. The results also show the optimal number of solar nodes is validate in the NHSND algorithm. On summary, NHSND meets the basic network coverage requirement and has a better energy consumption performance. The proper number of solar nodes balanced the network life time and the overall cost.

\section{REFERENCES}

[1] Akkaya, K., \& Younis, M. A survey on routing protocols for wireless sensor networks. Ad Hoc Networks, 2005, 3(8), 325-349. http://dx.doi.org/10.1016/j.adhoc.2003.09.010

[2] Gong, D., Yang, Y., \& Pan, Z. Energy-efficient clustering in lossy wireless sensor networks. Journal of Parallel \& Distributed Computing, 2013, 73(9), 1323-1336. http://dx.doi.org/10.1016/j.j pdc.2013.02.012

[3] Sun Y., Shen M., Zhou L., Xiong Y. \&Lin X. Simulation and realization of farmland wireless sensor networks nodes deployment. Transactions of the CSAE, 2010, 26(8), 211-215.

[4] Krishnanand, K. N., \&Ghose, D. Glowworm swarm optimization for simultaneous capture of multiple local optima of multimodal functions. Swarm Intelligence, 2009, 3(2), 87-124. http://dx.doi.org/10.1007/s11721-008-0021-5

[5] Yang, \&Xin She. Particle swarm optimization. AIAA 2002-1235. 43rd AIAA/AS-ME/ASCE/AHS/ASC, Structures, Structural Dynamics, and Materials Conference, April 22-25, 2002, 1(6), 9-40.

[6] Arostegui M A, Kadipasaoglu S N, Khumawala B M. An empirical comparison of Tabu Search, Simulated Annealing, and Genetic Algorithms for facilities location problems[J]. Bmc Research Notes, 2015, 8(1):1-9.

[7] Menga, L. I., Ding, D. R., \&Guo, T. L. Random deployment strategy of wireless sensor network node. Computer Engineering, 2012, 38(5), 99-101.

[8] Liu Hui, MengZhijun, Xu Min, \& Shang Yuanyuan. Sensor nodes deployment based on regular patterns in farmland environmental monitoring. Transactions of the Chinese Society of Agricultural Engineering, 2011, 27(8), 265-270(6).

[9] Sun Yuwen, ShenMingxia, Zhou Liang, et al. Simulation and realization of farmland wireless sensor networks nodes deployment[J]. Transactions of the CSAE, 2010, 26(8): 211-215.

[10] Heinzelman W R, Chandrakasan A, Balakrishnan H. Energyefficient communication protocol for wireless sensor networks[C]// Hawaii International Conference on System Sciences. IEEE, 2000:8020.

[11] Li, Q., Zhu, Q., \& Wang, M. Design of a distributed energyefficient clustering algorithm for heterogeneous wireless sensor networks. Computer Communications, 2006, 29(12), 2230-2237. http://dx.doi.org/10.1016/j.comcom.2006.02.017
[12] Buttyan, L., \&Hubaux, J. P. Enforcing Service Availability in Mobile Ad-Hoc WANs. Mobile and Ad Hoc Networking and Computing, 2000.MobiHOC. 2000 First Annual Workshop on (pp.87--96). IEEE. http://dx.doi.org/10.1109/mobhoc.2000.869 216

[13] Salami A F, Bello-Salau H, Anwar F, et al. A Novel Biased Energy Distribution (BED) Technique for Cluster-Based Routing in Wireless Sensor Networks[J]. International Journal on Smart Sensing \& Intelligent Systems, 2011, 4(2): 161 - 173.

[14] Ssu K F, Wang W T, Wu F K, et al. K-barrier coverage with a directional sensing model[J]. International Journal on Smart Sensing \& Intelligent Systems, 2009, 2(1): 75 - 93.

[15] Truong T T, Brown K N, Sreenan C J. Multi-objective hierarchical algorithms for restoring Wireless Sensor Network connectivity in known environments[J]. Ad Hoc Network, 2015, 33:190-208. http://dx.doi.org/10.1016/j.adhoc.2015.05.005

[16] Liu X. A Deployment Strategy for Multiple Types of Requirements in Wireless Sensor Networks[J]. IEEE Transactions on Cybernetics, 2015, 45(10):2364-2376. http://dx.doi.org/10.11 09/TCYB.2015.2443062

[17] Le Berre M, Rebai M, Hnaien F, et al. A Specific Heuristic Dedicated to a Coverage Tracking Bi-objective Problem for Wireless Sensor Deployment[J]. Wireless Personal Communications, 2015,84(3): 2187-2213. $\quad$ http://dx.doi.org/10.10 07/s11277-015-2548-2

[18] HONG Zhen, YU Li, ZHANG Gui-Jun. An Adaptive Distributed Clustering Routing Protocol for Wireless Sensor Networks[J]. Acta Automatica Sinica, 2011, 37(10):1197-1205.

[19] Davankov V A, Rogozhin S V, Kurganov A A. Analysis of Stochastic -Coverage and Connectivity in Sensor Networks With Boundary Deployment[J]. IEEE Transactions on Intelligent Transportation Systems, 2015, 16(4):1-11.

[20] Wu Huarui, Zhao Chunjiang, Zhang Haihui. Cluster head cycleswitching schemes for farmland wireless sensor networks[J]. Transactions of the CSAE, 2009, 25, (2): 170-174.

\section{AUTHORS}

Jianwei WU (corresponding author) is a senior engineer, presided over 10 government projects, published 32 papers, 15 patent applications. In-depth research and transformation for agricultural IOT, agricultural cultural creative and others. Selected outstanding young talents, science and technology nova plan of Beijing. Jianwei $\mathrm{Wu}$ is with the Beijing Research Center for Information Technology in Agriculture, Beijing Academy of Agriculture and Forestry Sciences, Beijing, China and the Beijing PAIDE Science and Technology Development Co. Ltd, Beijing, China (e-mail: wujw@nercita.org.cn).

Yisheng MIAO is an assistant researcher at National Engineering Research Center for Information Technology in Agriculture. His research interests include wireless sensor networks and intelligent systems in agriculture. He is with Beijing Research Center for Information Technology in Agriculture, Beijing Academy of Agriculture and Forestry Sciences, Beijing, China and with National Engineering Research Center for Information Technology in Agriculture, Beijing, China (e-mail: miaoys@nercita.org.cn).

This work was supported by the Beijing Municipal Science and Technology Project(Grants No. Z151100003215008), the National Natural Science Foundation of China (Grants No. NSFC-61271257), the National Natural Science Foundation of China (Grants No. NSFC-61471067), and the National Natural Science Foundation of China (Grants No. NSFC-61571051). Submitted, August, 19, 2016. Published as resubmitted by the authors on October, 20, 2016. 